\title{
Multimodal Classification using Feature Level Fusion and SVM
}

\author{
Priyanka Sharma \\ Department of Computer Science and Engineering \\ PEC University of Technology, \\ Chandigarh, India
}

\author{
Manvjeet Kaur \\ Department of Computer Science and Engineering \\ PEC University of Technology, \\ Chandigarh, India
}

\begin{abstract}
The use of biometrics in the field of enhancing security and authentication in sensitive systems is a rapidly evolving technology. The increasing attacks and decreasing security in unimodal systems have resulted in designing multimodal systems combining different biometric traits. A lot of research has already been done in designing multimodal systems with fusion at rank and match-score level using different classifiers such as Bayesian classifiers, LDA, ANNs and SVMs. In this research work, a multimodal system is designed by integrating face, fingerprint and palmprint based on feature level fusion. Each of the feature vectors are extracted independently using PCA and then fused together to perform classification using a multiclass SVM. The classification is performed on a set of test images taken from both the standard databases and live images captured in biometric lab.
\end{abstract}

\section{Keywords}

Multimodal biometric system, Feature level fusion, PCA, SVM.

\section{INTRODUCTION}

Biometrics, as the term suggests, is a measurement or identification of human beings based on their characteristics or biological traits. In the field of computer science, biometrics can be defined as the study of automating the authentication of an individual based on his physiological traits (i.e., face, fingerprint, iris, palm, etc.) or behavioral traits (typing rhythm, gait, voice, signature etc.)[2][10].

Biometric authentication came into existence when the security provided by the traditional methods of establishing a person's identity was found to be limited [1]. The traditional methods include either the knowledge-based identification systems such as passwords, personal identification number or token-based identification systems such as passport, driving license. These systems are not considered secured as they can be easily stolen, lost or shared. So the biometrics features which are unique for an individual are considered more reliable and natural in identifying the identity of an individual.

Now designing a biometric system using a single trait sometimes fails to provide accurate results because of its probabilistic nature. That is, if we collect multiple samples of the same biometric trait, every time it might not be same, e.g., no two photographs of a person's face are the same [2]. Also the unimodal biometric systems suffers from a variety of problems like noisy sensor data, non-universality, restricted degree of freedom, intra-class variations, spoof attacks and unacceptable error rates [3][4].
These problems of single trait biometric systems can be overcome by designing multimodal biometric systems which provides multiple evidence of the same feature. As described in [7], the multimodal biometrics systems provide a more secure environment with higher authentication accuracy by overcoming the limitations of individual biometric. A multibiometric system uses multiple sensors for acquiring data which further allows capturing of either multiple samples of the same biometric trait or multiple samples of more than one biometric trait [1]. There can be four scenarios of fusion in designing such systems [5][6]:

- $\quad$ Single biometric multiple sensors: here multiple sensors are used to capture multiple snapshots of the same biometric and then the result of all the sensors is combined for further processing.

- $\quad$ Single biometric multiple representation: here multiple instances of the same biometric are used for enrollment process (training process of a classifier). Then in the recognition module a single trait is captured and matched against several stored templates acquired earlier.

- Single biometric multiple matchers: Here different matching algorithms are used in the matching module and their results are then combined. Also here all the matching algorithms operate on the same representation of the single biometric trait.

- Multiple biometric multiple sensors: here multiple sensors are used to capture samples of multiple biometric characteristics and the result of each sensor is then fused together to provide as an input to the classifier. As the result of each sensor is from different domain, normalization technique is used before fusion.

Such systems are considered to be more reliable than unimodal biometric systems due to the presence of multiple, independent biometric features because it is very difficult for an intruder to forge all the features simultaneously. The key to form a successful multibiometric model is to decide and use an effective fusion scheme. Now the fusion in a multibiometric system is done by integrating information from multiple features using one of the following five different fusion techniques [5][6][8][9]:

- Sensor level: This fusion strategy requires the raw data to be acquired from multiple sensors which can be further processed and integrated to generate new data from which features can be extracted.

- Feature level: The information is extracted from the different sources and is further concatenated into a joint feature vector, which is then compared to an enrollment 
template (which itself is a joint feature vector stored in a database) and classification is performed accordingly.

- Match score level: This type of fusion creates the feature vectors independently for each modality and then compares them to the enrollment templates which are stored separately for each biometric trait during enrollment module. Based on the similarity of feature vector and the template, each subsystem calculates its own matching score value. These individual scores are finally combined to obtain a total score, which is then passed to the decision module.

- Rank level: This type of fusion is relevant in identification systems where each classifier associates a rank with every enrolled identity. Thus, fusion entails consolidating the multiple ranks associated with an identity and determining a new rank that would aid in establishing the final decision.

- Decision level: Here, a separate authentication decision is computed for each biometric trait which is then combined to result in a final vote. Fusion at this level is considered to be rigid compared to the other fusion schemes due to the availability of limited information.

The type of fusion scheme to be chosen highly depends on the type of application, the biometric traits and the level of fusion. The above described fusion schemes can be divided further into two distinct categories based on the level of fusion [9]. The sensor level and the feature level fusion come under prior to matching fusion and the rest three are part of after matching fusion category. It is believed that the biometric systems that perform fusion at an early stage are more effective than those which perform fusion at the later stage [5][9].

A lot of research has been done in this field of computer science. In 1995, Brunelli et al. [11] describes a multimodal biometric system based on acoustic and visual features where the classifier of both the traits have been integrated at rank level using HyperBF networks. The performance of this integrated system is shown to be superior to that of the acoustic and visual subsystems. Bigun et al. [12] developed a statistical model based on Bayesian statistics to integrate the information collected in form of speech (text-dependent) and face data of a user. Hong et al. [13] combined face recognition and fingerprint verification technique for developing automatic person identification system which operates in the identification mode with an admissible response time. The system overcomes the limitation offered by solely face recognition system or fingerprint verification system. Also, the proposed decision fusion scheme enables performance improvement by integrating multiple cues with different confidence measures.

In 2000, Dialog Communication Systems (DCS AG) developed a commercial multimodal product called BioID [14] that uses a model-based face classifier, a VQ-based voice classifier, and an optical-flowbased lip movement classifier for verifying persons. This integrated system outperforms the single-feature systems. Jain and Ross [15] improved the performance of a multimodal biometric system by learning user-specific parameters. User specific parameters include both the user specific thresholds and user specific weights. In their research the user-specific thresholds increased the system performance by $2 \%$ and the user-specific weights increased it by around $3 \%$.
In 1998, J. Kittler, M. Hatef, R. P. W. Duin, and J. Matas [16] developed a theoretical framework for combining classifiers that uses distinct representations of patterns. Here a comparison of various classifier combination schemes have been done experimentally and the sum-rule scheme outperforms the other combination schemes. Prabhakar and Jain [17] described a decision level fusion strategy which combines four different fingerprint matching algorithms and improved the system performance by $3 \%$. Also they showed that a combination of multiple impressions or multiple fingers improves the system performance by more than $4 \%$ and $5 \%$ respectively.

In 1997, U. Dieckmann, P. Plankensteiner, and T. Wagner [18] described a person authentication system SESAM which combines three different cues using sensor level fusion among which two of them are dynamic (sound of the voice and the lip motion) and the third one is static (it is the face image). Each feature is classified separately and then the results are combined to result a robust and reliable person authentication system. S. Ben-Yacoub, Y. Abdeljaoued, and E. Mayoraz [19] uses a combination face and speech to build a person identity authentication system. Here many binary classifiers (SVM, MLP, C4.5, LDA and Bayesian classifier) are compared on a large database. Among all the classifers, SVM and Bayesian showed better result than the rest.

In 2003, Fierrez-Aguilar and Ortega-Garcia [20] proposed a multibiometric system using score level fusion of a face , fingerprint and signature. The face verification system was based on a global appearance representation scheme, the fingerprint system based on minutiae, and the online signature verification system based on HMM modeling of temporal functions. In the same year, A. Kumar, D. C. M. Wong, H. C.Shen1, and A. K. Jain [21] presented a multimodal approach using palmprint and hand geometry, with fusion done at feature level by combining the feature vectors of both using concatenation operation, and the matching score level by using max rule.

Wang et al.[22] proposed a multimodal approach for a PCAbased face verification system and a key local variation-based iris verification system, with fusion at the match score level by using unweighted and weighted sum rules, LDA and the neural networks. In 2004, K. A. Toh, X. D. Jiang, and W. Y. Yau [23] developed a multimodal system using hand geometry, fingerprint, and voice biometric with weightedsumrule-based match-score-level fusion. A reduced multivariate polynomial model was introduced to overcome the tedious recursive learning problem.

In 2005, Snelick et al. [24] developed a multimodal using fusion of face and fingerprint, applying the fusion methods at the score level. Seven score normalization techniques (minmax, $z$-score, tanh, adaptive, two quadrics, logistic, and quadric-line-quadric) and five fusion techniques on the normalized scores (simple sum, $\min$ score, $\max$ score, matcher weighting, and user weighting) were used in this study for testing. All the normalization techniques with fusion combinations outperformed any monomodal systems tested in this study except for the min-score fusion approach. In the same year, Jain et al. [25] proposed a multimodal approach using face, fingerprint, and hand geometry, where the fusion done at match score level. Various score normalization techniques and fusion rules are discussed and compared. The experiments were conducted on a database of 100 users which indicates that the use of min-max, z-score, and tanh normalization schemes followed by a simple sum of scores 
fusion method results in better recognition performance compared to other methods.

\section{PROPOSED METHOD}

As mentioned in the literature review above, the unimodal biometric models possess various limitations which are overcome by using a multimodal biometric system. This paper presents a multimodal system using the most frequently used biometric traits, i.e., face, fingerprint and palmprint. As all these three traits have different domain, the proposed criterion makes use of Principal Component Analysis (PCA) algorithm to extract important features from the input images of all these three features and resulting them into a common domain. Also, PCA acts as the dimensionality reduction algorithm which in turn reduces the search space for the classifier.

The result of PCA algorithm consists of three feature matrices, i.e. each of face, fingerprint and palmprint which are further fused together using feature level fusion to result in a single resultant matrix. This resultant matrix is then fed as an input to the Support Vector Machine (SVM) classifier which uses it for both training and testing. The result of SVM gives the accuracy with which the test images are classified. If the test image is a genuine image present in the database, SVM gives the output as 1 , else 0 .

The design of the proposed system is shown below:

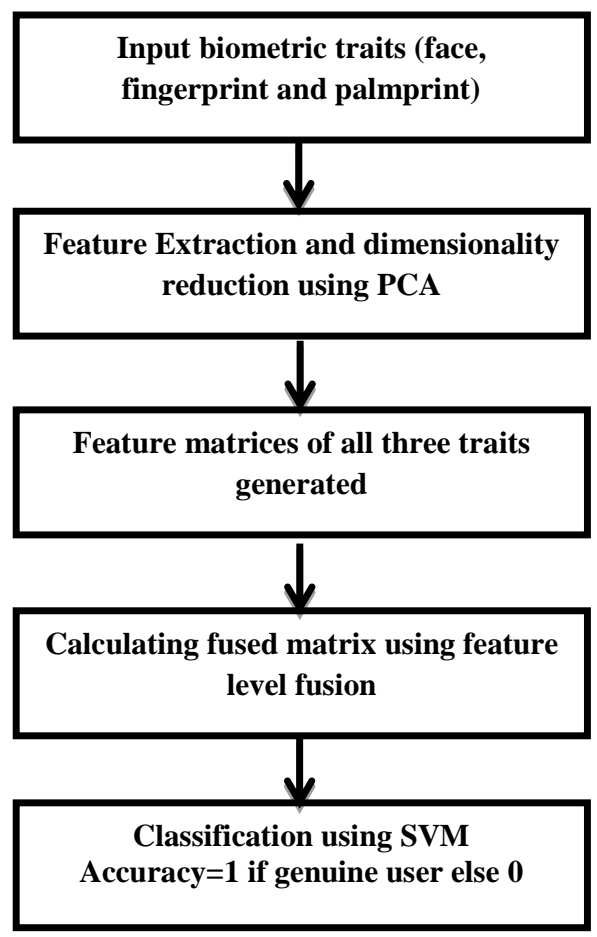

Figure1. Work Flow

\subsection{Feature Extraction and Dimensionality Reduction using PCA}

PCA is a useful statistical technique that has its application in various fields of face recognition and image compression [44]. It is a common technique used for dimensionality reduction in data of high dimension [35]. It is a way of identifying patterns in data, and expressing the data in such a way that highlights their similarities and difference. Basically PCA algorithm is used only for face recognition systems, but now it has also been used in fingerprint verification and palmprint recognition [45][46]. In the research work, it has been applied to all the three traits used in the fusion (face, fingerprint and palmprint). In PCA, the following are the steps which are needed to perform feature extraction and dimensionality reduction on all the input images (both training and testing images)[35][37]:

- $\quad$ The coloured image samples are taken and converted to grey scale images.

- $\quad$ Resize the image

- Transform the 2D matrix to 1D column vector.

- Calculate the mean vector $\mathrm{R}$ of data vectors in matrix $\mathrm{S}$

- Normalize the vector of data matrix $S$ with subtracting by the mean vector $\mathrm{R}$ and stored in $\mathrm{T}$ variable

- Calculate the covariance matrix of the column vectors: as $\mathrm{CV}=\mathrm{T} * \mathrm{~T}$.

- Compute the eigen values and corresponding eigenvectors as $\mathrm{W}=\mathrm{eig}(\mathrm{cv})$

- $\quad$ Get the $\mathrm{P}$ by projecting the data matrix $\mathrm{X}$ onto the eigen space $\mathrm{P}=\mathrm{W}^{\mathrm{T}} * \mathrm{X}$

The figure below shows some of the eigen-features of different individuals where each column contains eigen-face, eigen-finger and eigen-palm of same individual. The eigenfeatures are the principal components resulted from the PCA algorithm which helps in simplifying the classification problem by reducing the search space for the classifier.

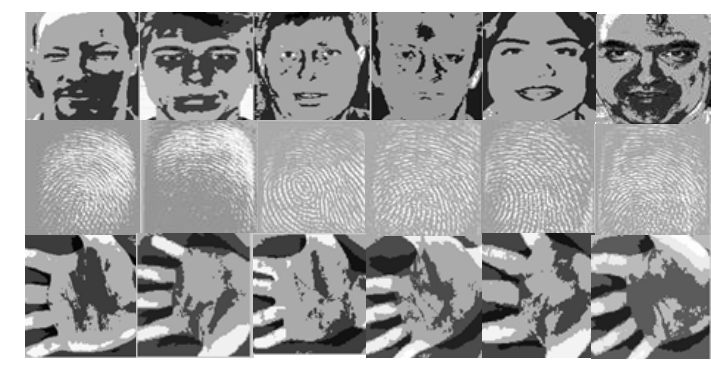

Figure2. Output of PCA algorithm

All these feature values are then fused and provided as an input to the classifier for training as well as testing purposes.

\subsection{Feature Level Fusion}

Fusion at the feature level involves the integration of feature sets corresponding to multiple modalities. As the feature set contains richer information about the raw biometric data than the match score or the final decision level, fusion at feature level is considered better than the other fusion strategies. First of all, the features are extracted from multiple biometric traits. Before extracting features some preprocessing steps to be done for the image enhancement. Features from the biometric traits are represented in the form of vectors.

Feature level fusion has some limitations also and these are given below:

- Incompatible feature sets

- The relation between the feature vectors of different biometric traits is not known

- $\quad$ significant more complex matchers might be required 
In this research work features from all three biometric traits are extracted using the same PCA algorithm which resulted in the feature sets of same domain. Thus all the three feature sets are compatible with each other and hence fused easily using simple concatenation function of matlab.

\subsection{Support Vector Machine (SVM) Classifier}

SVM is a pattern classification algorithm developed by Vapnik and Cortes [27] based on statistical learning theory. SVM has many advantages in solving small classification samples which are nonlinear and also high dimensional problems in pattern recognition. Unlike traditional neural network which are based on Empirical Risk Minimization (ERM) principle, SVM are based on Structural Risk Minimization (SRM) principle, which has been shown to be superior to ERM by Steve R. Gunn and Martin Brown in 1997. SRM minimizes an upper bound on the expected risk in contrast to ERM which minimizes the error on the training data [29]. It is the property which equips SVM with a greater ability to generalize itself to solve various classification problems, which is the basic goal in statistical learning. Initially SVM was developed only for classification problems but later on, it has extended to regression also [28]. In comparison to the other classifiers, SVM has provided better performance [30][31][32][33][34]. In this paper, the classification is being performed using SVM.

\subsubsection{Basics of SVM}

The basic SVM is a two class SVM which can separate the data into two classes by finding a hyperplane between them. There can be multiple hyper planes present but the goal of SVM is to find the optimal separating hyperplane which error-freely separate the data into two classes and also maximizes the margin between the two classes (maximizes the distance between it and the nearest data point of each class called support vectors)[29][35][37].

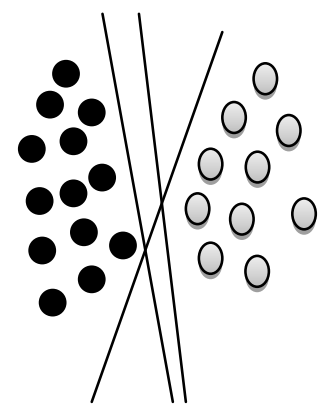

Figure 3. Linearly separable data with multiple arbitrary[37]

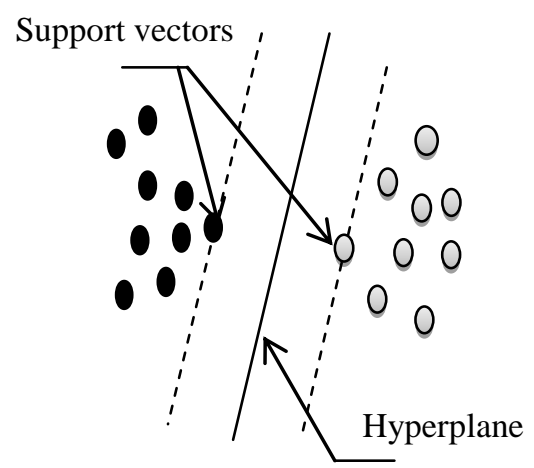

Figure4. Linearly separable data with optimal hyperplane [37]

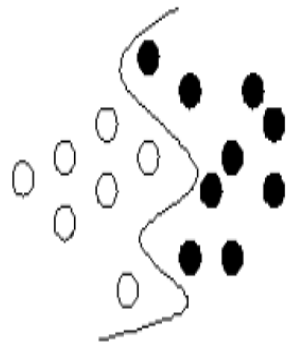

Figure 5. Linearly inseparable data[37]

To find an optimal hyperplane, SVM has kernel functions that act as a separating function. The kernel functions are an integral part of SVM which separates the input data into two classes involving minimum number of support vectors [38]. There are four types of kernel functions which are as follows [39]:

- $\quad$ Linear: $K\left(x_{i}, x_{j}\right)=x_{i}^{T} x_{j}$

- Polynomial: $K\left(x_{i}, x_{j}\right)=\left(\gamma x_{i}^{T} x_{j}+r\right)^{d}, \gamma>0$

- radial basis function (RBF):K( $\left.x_{i}, x_{j}\right)=\exp \left(-\gamma \| x_{i}-\right.$ $\left.x_{j} \|^{2}\right), \gamma>0$

- $\quad$ sigmoid: $K\left(x_{i}, x_{j}\right)=\tanh \left(\gamma x_{i}^{T} x_{j}+r\right)$

Here all these parameters, i.e., $\gamma, \mathrm{r}$ and $\mathrm{d}$ are the kernel parameters.

The selection of kernel function depends on the type of classification problem. Mostly RBF is a reasonable choice because of the following reasons [39]:

- RBF non-linearly maps the samples to a high dimensional space, i.e., it can handle the cases where the relation between class labels and its attributes is nonlinear.

- The number of hyperparameters which affect the complexity of model are less for RBF kernel. During model selection, only value of cost $(\mathrm{C})$ and gamma $(\gamma)$ have to be predicted and chosen.

In this research paper, RBF is chosen as the kernel function because of the increase in accuracy that this kernel provides over the other kernels (as shown using the following graphs).

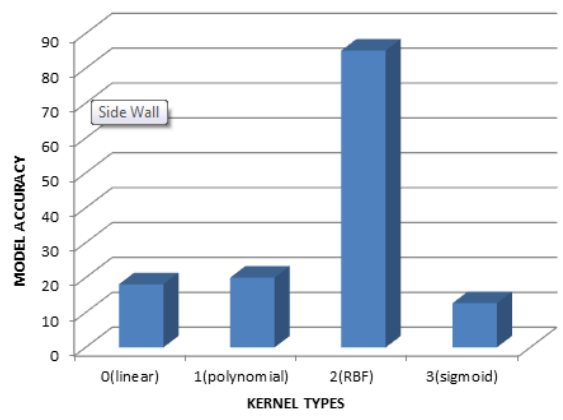

Figure 6. Model accuracy with all the kernel types 


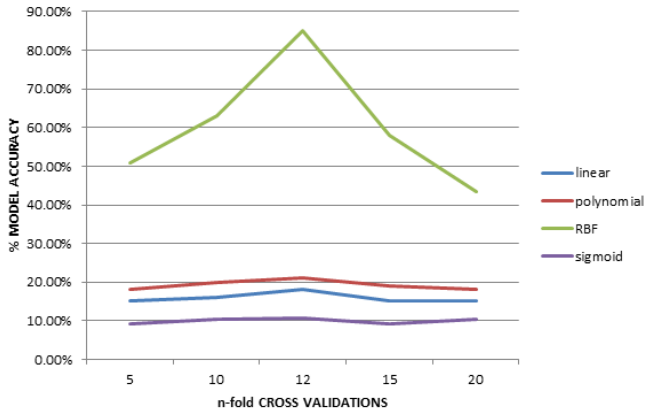

Figure 7. Quantitative evaluation of all kernels with the cross validation

The process of finding the best values of $\mathrm{C}$ and $\gamma$ for RBF kernel is formally known as cross-validation. To perform cross-validation, grid search is recommended [39].

\subsubsection{Multiclass Classification}

Basically SVM is a used for binary class problem. But now it can be extended to support multi-class problems. To convert a binary class SVM to multi-class SVM, there are two strategies: one-against-one strategy to classify between each pair and one-against-rest strategy to classify between each class and all the remaining. Both the schemes can be used to make a multiclass SVM. To build a k-class classifier, oneagainst-one scheme requires $\mathrm{k}(\mathrm{k}-1) / 2$ binary SVMs while a one-against-rest strategy needs k SVMs. Although both the strategies helps in making a multi-class classifier, oneagainst-one is preferred [40][41][42].

There are various software's present which helps in implementation of multi-class SVM among which LIBSVM [36][39]is used in this paper because this software provides interface for multiple languages including matlab. Also it supports multiclass SVM's implementation with graphic interface [43].

In this research work, the following steps have been performed for classification of data into 10 different classes:

- The output of the feature level fusion algorithm is first converted to LIBSVM format.

- Then simple scaling is performed on the data to keep all the feature values between $\{-1,1\}$.

- $\quad$ Now to design a SVM model, RBF kernel is selected.

- After this grid search in cross validation is done to find the best parameters values $(\mathrm{C}$ and $\gamma)$.

- These best parameter values are then used to train the model.

- After training is performed, testing is done using the test data.

In a biometric system, the performance is usually evaluated in two different cases: verification and identification. In the 'verification' mode, the system is expected to check the user claimed identity providing a binary answer in the form of 'accepted' or 'rejected'. In the 'identification' mode, the system is expected to identify the genuine user from a collection of templates stored in a database[26].

In this research work, the performance of multimodal biometric system is computed verification in mode. In this, the system provides the output as accepted or rejected. It gives accuracy as $100 \%$ for accepted test image if it is present in our database and $0 \%$ if it is not present in the database.

The test set consists of 100 different images. The result of SVM classification results in an accuracy $\sim=95 \%$ while the sensitivity(True positive rate) $\sim=94 \%$ and the specificity(True negative rate) is $\sim=96 \%$ for the static images of the standard databases (frontal face images collected by Markus Weber at California Institute of Technology, fingerprint taken from DB3_(UPEK)and palm images taken from CASIA palmprint database). In this case, FAR $\sim=4 \%$ and FRR $=6 \%$ where FAR is False acceptance rate and FRR is False rejection rate.

The SVM multiclass implementation is also tested on the live images collected at our site using Cross match's Verifier 300 LC scanner and an integrated face recognition camera in Apple Macintosh machine. The test set for live database is taken of 50 images. Its accuracy comes out to be $\sim=92 \%$ with sensitivity $\sim=88 \%$ and specificity $\sim=96 \%$. Here the FAR = $4 \%$ and FRR = $12 \%$.

Some snapshots of the GUI for this research work are follows:

- GUI with test input from static standard database

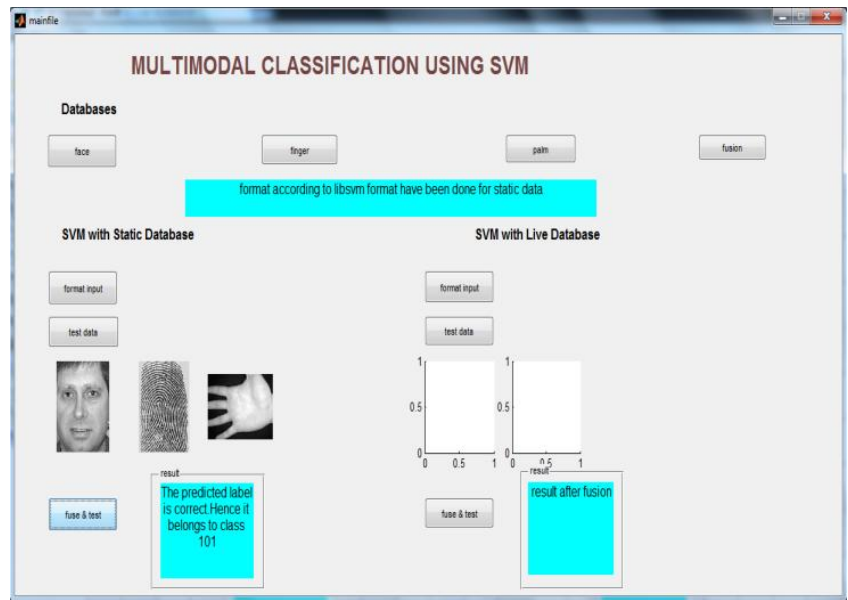

- GUI taking test input from live database (taking fusion of only face and fingerprint

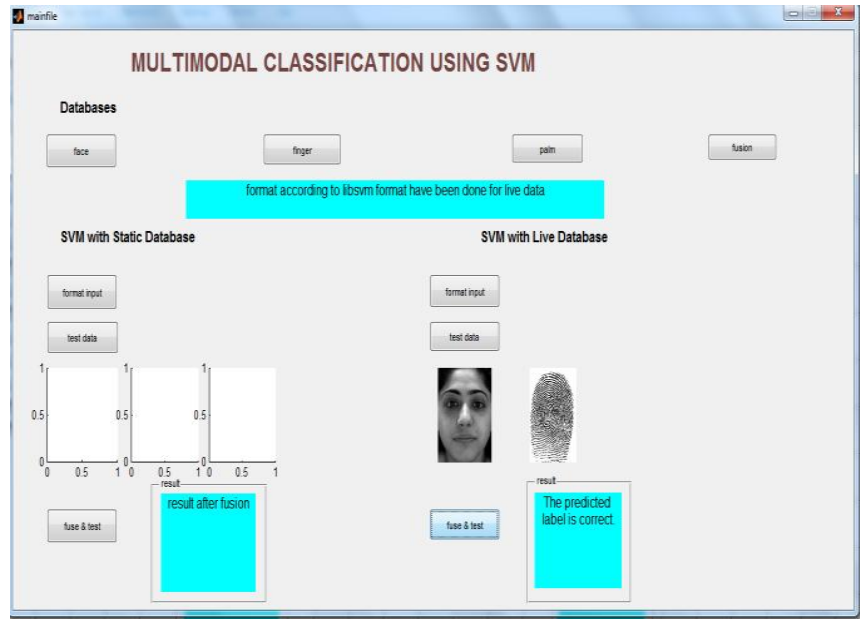




\section{CONCLUSION}

Providing authentication and security using multimodal systems is a promising technique which combines the features of multiple modalities and is proved better than using single traits. This research paper presents a multimodal system using face, fingerprint and palmprint. The combined feature vector is experimentally tested and compared with individual trait feature vector and the classification results are better for combined vector. Also classification using SVM is compared with classification using simple feed forward neural network and the results of SVM are much better than NN. Also this research paper discusses feature level fusion with SVM classifier which is a newly discussed technique in designing multimodal systems.

\section{REFERENCES}

[1] Savitri B. Patil, "A Study of Biometric, Multimodal Biometric Systems: Fusion Techniques, Applications and Challenges", IJCST Vol. 3, Issue 1, Jan- March 2012.

[2] P. Jonathon Phillips and Elaine M. Newton, "Biometric Systems: The Rubber Meets the Road", Proceedings of the IEEE, Vol. 97, No. 5, May 2009.

[3] Arun Ross and Anil K. Jain, "Multimodal Biometrics: An Overview", Appeared in Proc. of 12th European Signal Processing Conference (EUSIPCO), (Vienna, Austria), pp. 1221-1224, September 2004.

[4] V C Subbarayudu and Munaga V N K Prasad, "Multimodal Biometric System", IEEE computer society, 2008.

[5] Anil K. Jain, Arun Ross and Salil Prabhakar, "An Introduction to Biometric Recognition", Appeared in IEEE Transactions on Circuits and Systems for Video Technology, Special Issue on Image- and Video-Based Biometrics, Vol. 14, No. 1, January 2004.

[6] Arun Ross and Anil Jain, "Information Fusion in Biometrics", Pattern Recognition Letters 24 (2003) 2115-2125, Elsevier Science, 2003.

[7] Lin Liu, Xiao-Feng GU, Jian-Ping Li, Jie Lin, Jin-Xin Shi, Yuan-Yuan Huang, "Research on Data Fusion of Multiple Biometric Features”, IEEE 2009.

[8] Arun Rossa and Rohin Govindarajan, "Feature Level Fusion Using Hand and Face Biometrics", Appeared in Proceedings of SPIE Conference of Biometric Technology for Human Identification II, Vol. 5779,pp. 196-204, USA,march 2005.

[9] Md. Maruf Monwar and Marina L. Gavrilova, "Multimodal Biometric System Using Rank-Level Fusion Approach", IEEE Transactions on Systems on Man and Cybernetics-Part B Cybernetics, Vol. 39, No. 4, August 2009.

[10] A. K. Jain, A. Ross, and S. Pankanti, "Biometrics: A tool for information security," IEEE Trans. Inf. Forensics Security, vol. 1, no. 2, pp. 125-143, Jun. 2006

[11] R. Brunelli and D. Falavigna,"Person Identification Using Multiple Cues", IEEE Trans. On Pattern Analysis and Machine Intelligence, Vol. 12, No. 10, pp. 955-966, Oct 1995
[12] E. S. Bigun, J. Bigun, B. Duc, and S. Fischer, "Expert Conciliation for Multimodal Person Authentication Systems using Bayesian Statistics", Proc. International Conference on Audio and Video-Based Biometric Person Authentication (AVBPA), pp. 291-300, CransMontana, Switzerland, March 1997.

[13] L. Hong and A. K. Jain, "Integrating Faces and Fingerprints for Personal Identification", IEEE Trans. on Pattern Analysis and Machine Intelligence, Vol. 20, No. 12, pp. 1295-1307, Dec 1998.

[14] R. W. Frischholz and U. Dieckmann, "Bioid: A Multimodal Biometric Identification System", IEEE Computer, Vol. 33, No. 2, pp. 64-68, 2000.

[15] A. K. Jain and A. Ross, "Learning User-specific Parameters in a Multibiometric System", Proc. International Conference on Image Processing (ICIP), Rochester, New York, September 22-25, 2002.

[16] J. Kittler, M. Hatef, R. P. W. Duin, and J. Matas, “On Combining Classifiers", IEEE Trans. on Pattern Analysis and Machine Intelligence, Vol. 20, No. 3, pp. 226-239, Mar 1998.

[17] S. Prabhakar and A. K. Jain, "Decision-level Fusion in Fingerprint Verification", Pattern Recognition, Vol. 35, No. 4, pp. 861-874, 2002

[18] U. Dieckmann, P. Plankensteiner, and T. Wagner, "Sesam: A Biometric Person Identification System Using Sensor Fusion", Pattern Recognition Letters, Vol. 18, No. 9, pp. 827-833, 1997.

[19] S. Ben-Yacoub, Y. Abdeljaoued, and E. Mayoraz, "Fusion of Face and Speech Data for Person Identity Verification”, Research Paper IDIAP-RR 99-03, IDIAP, CP 592, 1920 Martigny, Switzerland, Jan 1999.

[20] J. Fierrez-Aguilar, J. Ortega-Garcia, D. Garcia-Romero, and J. Gonzalez-Rodriguez, "A comparative evaluation of fusion strategies for multimodal biometric verification," in Proc. 4th Int. Conf. Audio- Video-Based Biometric Person Authentication, J. Kittler and M. Nixon, Eds., 2003,vol. LNCS 2688, pp. 830-837.

[21] A. Kumar, D. C. M. Wong, H. C.Shen1, and A. K. Jain, "Personal verification using palmprint and hand geometry biometric," in Proc. 4th Int. Conf. AudioVideo-Based Biometric Person Authentication, J. Kittler and M. Nixon, Eds., 2003, vol. LNCS 2668, pp. 668678.

[22] T. Wang, T. Tan, and A. K. Jain, "Combining face and iris biometrics for identity verification," in Proc. 4th Int. Conf. Audio- Video-Based Biometric Person Authentication, J. Kittler and M. Nixon, Eds., 2003, vol. LNCS 2688, pp. 805-813.

[23] K. A. Toh, X. D. Jiang, and W. Y. Yau, "Exploiting global and local decisions for multi-modal biometrics verification," IEEE Trans. Signal Process., vol. 52, no. 10, pp. 3059-3072, Oct. 2004.

[24] R. Snelick, U. Uludag, A. Mink, M. Indovina, and A. K Jain, "Large scale evaluation of multimodal biometric authentication using state-of the-art systems," IEEE Trans. Pattern Anal. Mach. Intelligence, vol. 27, no. 3, pp. 450-455, Mar. 2005 
[25] A. K. Jain, K. Nandakumar, and A. Ross, "Score normalization in multimodal biometric systems," Pattern Recognition, vol. 38, no. 12, pp. 2270-2285, 2005.

[26] Fakhreddine Karray, Jamil Abou Saleh, Mo Nours Arab and Milad Alemzadeh, "Multi ModalBiometric Systems: A State of the Art Survey".

[27] V. Vapnik, "The Nature of Statistical Learning Theory", Springer, N.Y., 1995. ISBN 0-387-94559-8.

[28] V. Vapnik., "Statistical Learning Theory", Springer, N.Y., 1998.

[29] S.R. Gunn, M. Brown, and K.M. Bossley, "Network performance assessment for neurofuzzy data modeling", In X. Liu, P. Cohen, and M. Berthold, editors, Intelligent Data Analysis, volume 1208 of Lecture Notes in Computer Science, pages 313-323, 1997.

[30] Morris, C.W. and Autret, A, "Support vector machines for identifying organisms - a comparison with strongly partitioned radial basis function networks", Ecological Modeling, Vol. 146, pp.57-67, 2001.

[31] Cai, Y-D. and Lin, X-J, "Prediction of protein structural classes by support vector machines", Computers and Chemistry, Vol. 26, pp.293-296, 2001.

[32] Juan-Manuel Ramirez-Cortes, Pilar Gomez-Gil, Vicente Alarcon- Aquino, David Baez-Lopez, Rogerio EnriquezCaldera, "A Biometric System Based on Neural Networks and SVM Using Morphological Feature Extraction from Hand-Shape Images", INFORMATICA, 2011, Vol. 22, No. 2, 225-240 225.

[33] Sandhya Arora. Debotosh Bhattacharjee, Mita Nasipuri, L. Malik , M. Kundu and D. K. Basu , "Performance Comparison of SVM and ANN for Handwritten Devnagari Character Recognition”, IJCSI International Journal of Computer Science Issues, Vol. 7, Issue 3, May 2010.

[34] M. Saberi, A. Azadeh, A. Nourmohammadzadeh and P. Pazhoheshfar, "Comparing performance and robustness of SVM and ANN for fault diagnosis in a centrifugal pump", 19th International Congress on Modelling and Simulation, Perth, Australia, 12-16 December 2011.
[35] Chengliang Wang, Libin Lan, Yuwei Zhang, Minjie Gu, "Face Recognition Based on Principle Component Analysis and Support Vector Machine”, 2011 IEEE.

[36] Chih-Chung Chang, Chih-Jen Lin, LIBSVM: a library for support vector machines, 2001. http://www.csie.ntu.edu.tw/ cjlin/libsvm.

[37] Md. Omar Faruqe, Md. Al Mehedi Hasan, "Face Recognition Using PCA and SVM".

[38] D. Boolchandani, and Vineet Sahula, "Exploring Efficient Kernel Functions for Support

[39] Vector Machine Based Feasibility Models for Analog Circuits", International Journal of Design, Analysis and tools for Circuits and Systems, Vol. 1, No. 1, June 2011.

[40] Chih-Wei Hsu, Chih-Chung Chang, and Chih-Jen Lin, "A Practical Guide to Support Vector Classification".

[41] M. Pontil and A. Verri, "Support vector machines for 3-d object recognition",IEEE Trans. on Pattern Analysis and Machine Intelligence, 20:637-646, 1998.

[42] Chih-Wei Hsu and Chih-Jen Lin, "A Comparison of Methods for Multiclass Support Vector Machines", IEEE Transactions on Neural networks, Vol. 13, No. 2, March 2002.

[43] www.kernel-machines.org

[44] Lindsay I Smith, "A tutorial on Principal Components Analysis", February 26, 2002

[45] Nirosha Joshitha J, R. Medona Selin, “Image Fusion using PCA in Multifeature Based Palmprint Recognition", International Journal of Soft Computing and Engineering (IJSCE) ISSN: 2231-2307, Volume-2, Issue-2, May 2012.

[46] Ching-tang Heieh, Shys-Rong Shyu, "Principal Component Analysis for Minutiae Verification on Fingerprint Image", Proceedings of the 7th WSEAS International Conference on Multimedia Systems \& Signal Processing, Hangzhou, China, April 15-17, 2007. 